\title{
Switching Model Predictive Attitude Control for a Quadrotor Helicopter subject to Atmospheric
}

\author{
Disturbances
}

\author{
Kostas Alexis a , George Nikolakopoulos ${ }^{\mathrm{b}, *}$ and Anthony Tzes ${ }^{\mathrm{a}}$ \\ ${ }^{a}$ Electrical and Computer Engineering Department, \\ University of Patras, \\ Rio 26500, GREECE ${ }^{\mathrm{a}}$ \\ ${ }^{\mathrm{b}}$ Computer Science, Electrical and Space Engineering Department, \\ Lulea University of Technology, \\ Lulea SE-97753, SWEDEN ${ }^{\mathrm{b}}$
}

\begin{abstract}
In this article a Switching Model Predictive Attitude Controller for an Unmanned quadrotor Helicopter subject to atmospheric disturbances is presented. The proposed control scheme is computed based on a Piecewise Affine (PWA) model of the quadrotor's attitude dynamics, where the effects of the atmospheric turbulence are taken into consideration as additive disturbances. The switchings among the PWA models are ruled by the rate of the rotation angles and for each PWA system a corresponding model predictive controller is computed. The suggested algorithm is verified in experimental studies in the execution of sudden maneuvers subject to forcible wind disturbances. The quadrotor rejects the induced wind-disturbances while performing accurate attitude tracking.
\end{abstract}

Key words: Unmanned Quadrotor Helicopter, Model Predictive Control, Switching Control, Atmospheric Disturbances. 


\section{Introduction}

Recent innovative designs of Vertical Take-Off and Landing (VTOL) Unmanned Aerial Vehicles (UAVs), have generated great interest in the control scientific community (Tayebi and McGilvray (2006); Kondak et al. (2007)). Within the family of the VTOL UAVs, Unmanned Quadrotor Helicopters (Hoffmann et al. (2007)) that base their operation in the appropriate control of four rotors, have received a growing attention, mainly due to their capability to outperform most of other types of helicopters on the issues of maneuverability, survivability, simplicity of mechanics and increased payloads (Bouabdallah et al. (2007)).

The quadrotors' hovering ability makes possible an extended area of applications for which fixed wing aircrafts are not suitable. Examples include forest fire surveillance (Merino et al. (2005); Alexis et al. (2009)), inspection of buildings and bridges (Metni and Hamel (2007)), wildfire monitoring, and military applications. During these missions precise trajectory tracking combined with effective disturbance attenuation is required. Quadrotors have several advantages compared to other rotorcraft designs including: a) the simplicity of their mechanical structure, b) the use of four small propellers resulting in a more fault-tolerant mechanical design capable of aggressive maneuvers at low altitude, c) good maneuverability and d) increased payload (Bouabdallah et al. (2007)).

The quadrotor's model is highly non-linear with certain parameters being depen-

\footnotetext{
* Corresponding Author

Email address: george.nikolakopoulos@ltu.se (George Nikolakopoulos).
} 
dent on the characteristics of the flight (Bouabdallah and Siegwart (2007)). Moreover, during flights in low-altitudes, quadrotors are prone to sudden wind-gusts that can significantly affect their flight performance and even cause instability. Therefore the development of specialized controllers, that could take under consideration the quadrotor's modeling non-linearities and the uncertainties while reacting to such sudden wind-gusts is desired.

In the relevant literature of quadrotors, the problem of control design has been addressed by a large number of publications that mainly focus in the following areas: a) PID control and LQ-Regulation in (Bouabdallah et al. (2004)), b) Sliding Mode control (Waslander et al. (2005)), c) Backstepping control (Bouabdallah and Siegwart (2007)), d) Integral predictive-nonlinear $H_{\infty}$ control (Raffo et al. (2010)), e) Constrained Finite Time Optimal Control Scheme (Alexis et al. (2010a,b)), and f) bounded control (Guerrero-Castellanos et al. (2011)). Moreover in the existing literature of rotorcrafts, certain simulation studies have focused on the effects of the environmental disturbances (Costello (1992); Yang et al. (2009)); in general an experimental verification is needed to validate the efficiency of the noted efforts.

On the effect of environmental disturbances to the performance of rotorcrafts, the majority of the recent published work in the control-community has focused on theoretical and simulation studies (Costello (1992); Yang et al. (2009); Perhinsch (1998); Martini et al. (2009)) with very few experimental articles focusing in the area of disturbance rejection. Extensive advanced aerodynamic models have recently appeared in the area of quadrotors (Pounds et al. (2010); Bristeau et al. (2009); Pounds et al. (2006)) with a direct implication on the control performance.

The work presented in this article focuses on the problem of controlling the attitude of a quadrotor in the presence of external wind-gust disturbances. The novel contri- 
butions of the proposed control design scheme are: a) the presentation of an optimal control design approach for the attitude control of a quadrotor, and b) the experimental application and evaluation of the suggested control scheme in a prototype quadrotor under a wind-gust disturbance.

The controller relies on the principle of Model Predictive Control (MPC) based on a Piecewise Affine (PWA) model description of the quadrotor's linearized model for a certain motion. In this approach the wind-turbulence affects the nominal system and is taken under consideration in the system model description as an affine term with an a priori known worst case bound.

Model predictive control determines the control input via a receding horizon optimal control approach based on the open-loop model of the quadrotor. The main appeal of MPC is the ability to: a) enforce pointwise-in-time constraints, b) count for additive disturbances at the phase of system modeling, while c) providing the control designer with the direct capability to shape the transient response by adjusting the weights in the objective function being minimized. These attributes are quite important in accurate aggressive maneuvers under wind-gusts of rotorcrafts in general (Alexis et al. (2010c)) and quadrotors in particular.

This article is organized as follows. In Section 2 the quadrotor's dynamics are presented while in Section 3 the mathematical formulation of the novel control design scheme based on a Switching variation of a MPC is analyzed. In Section 4 experimental studies, from multiple test-cases, are presented that prove the efficacy of the proposed scheme in the absence/presence of wind disturbances and in comparison with other controllers. Finally in Section 5, the conclusions are drawn. 


\section{Quadrotor's Dynamics}

The model of the quadrotor utilized in this work, assumes that the structure is rigid and symmetrical, the center of gravity and the body fixed frame origin coincide, the propellers are rigid and the thrust and drag forces are proportional to the square of propeller's speed. The quadrotor, as a rigid body, is characterized by the linked body fixed frame characterized by $\mathscr{B}=\left\{\mathbf{B}_{1}, \mathbf{B}_{2}, \mathbf{B}_{3}\right\}$, where the $\mathbf{B}_{1}$ axis is in the helicopter normal flight direction, $\mathbf{B}_{2}$ is orthogonal to $\mathbf{B}_{1}$ and positive to starboard in the horizontal plane, whereas $\mathbf{B}_{3}$ is oriented in the ascendant sense and orthogonal to the plane $B_{1} O B_{2}$, as presented in Figure 1 .

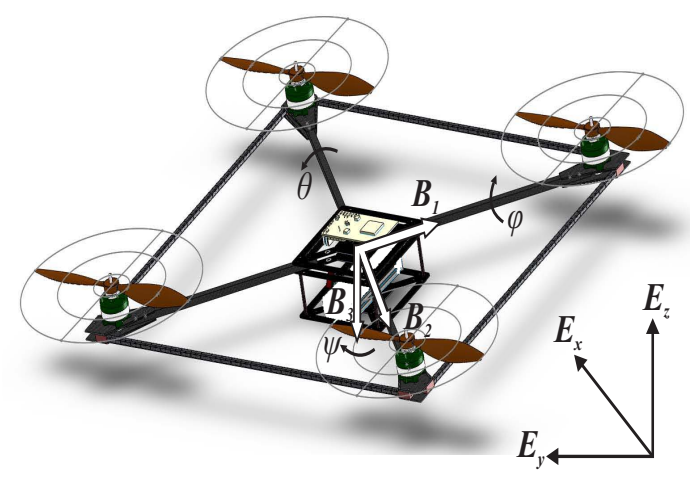

Fig. 1. Quadrotor helicopter configuration frame system

The aerodynamic forces and moments acting on the quadrotor during a hovering flight segment correspond to the thrust $(\tau)$, hub forces $(\mathbf{h})$ and drag moment $\left(\mathbf{m}_{d}\right)$ due to vertical, horizontal and aerodynamic forces respectively, followed by the rolling moment $\left(\mathbf{m}_{r}\right)$ related to the integration over the entire rotor of the lift of each section, acting at a given radius. An extended formulation of these forces and moments can be found at (Bouabdallah and Siegwart (2007)).

Let a) the translation-vector movement of the quadrotor's center of mass be $\xi=$ $\left.[x, y, z]^{T}, \mathrm{~b}\right)$ the rotations of the quadrotor with respect to the ground be $\eta=$ 
$[\phi, \theta, \psi]^{T}$, then the system's dynamics can be formulated as:

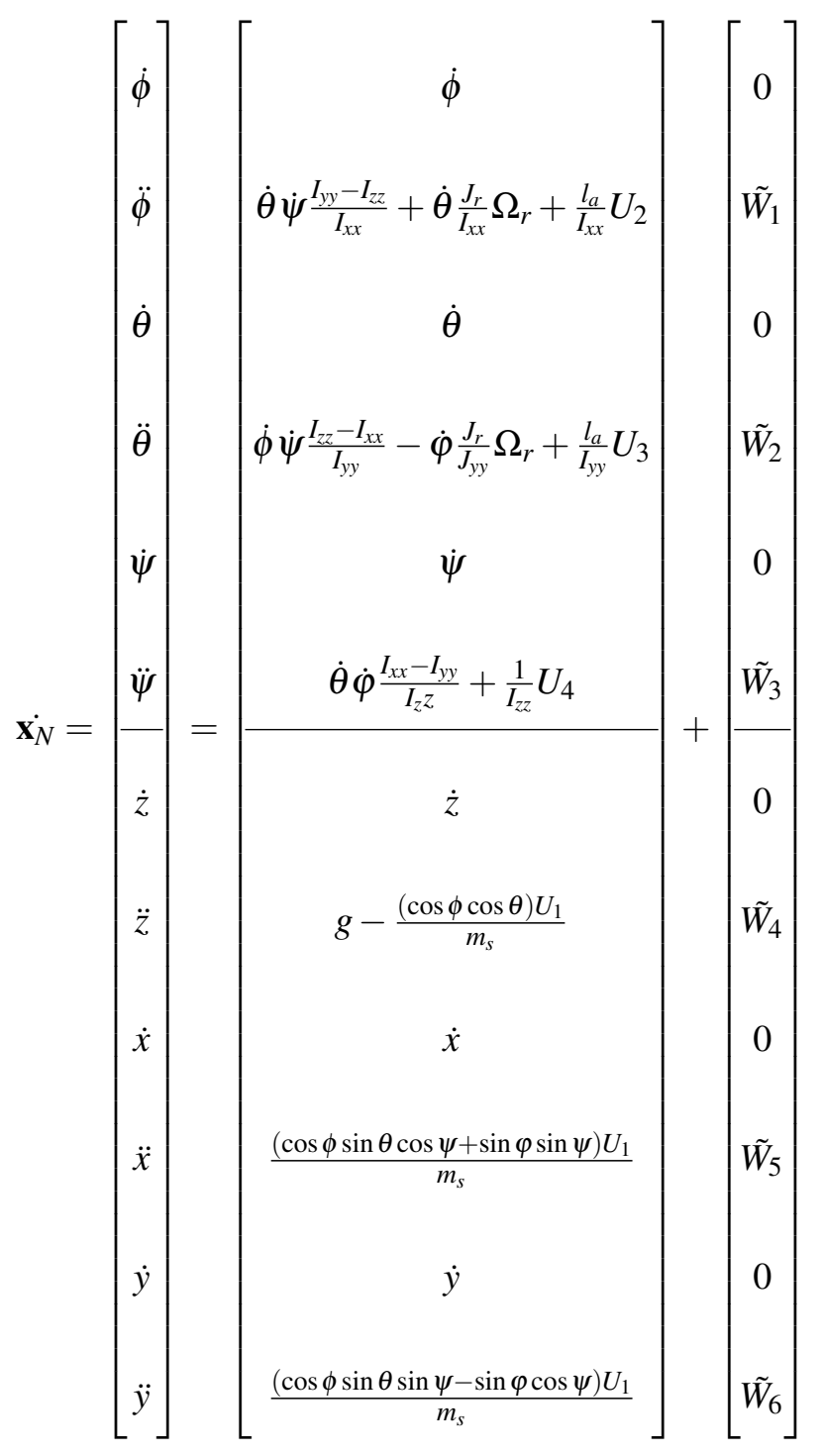

where $m_{s}$ is the mass of the system, $g$ the gravity acceleration, $\tilde{W}_{i}, i=1, \ldots, 6$ terms represent the effect of the wind gusts on the quadrotor's angular and translational accelerations in the form of additive disturbances, and $U_{i}, i=1, \ldots, 4$ correspond to the control actions that are dependent on the four motors' angular velocities $\Omega_{i}, i=1, \ldots, 4$; the control vector $\mathbf{u}_{N}$ is formed by the introduction of one auxiliary variable $\Omega_{r}$, as $\mathbf{u}_{N}=\left[U_{1}, \ldots, U_{4}, \Omega_{r}\right]^{T}$.

The control actions are calculated as: 


$$
\mathbf{u}_{N}=\left[\begin{array}{c}
U_{1} \\
U_{2} \\
U_{3} \\
U_{4} \\
\Omega_{r}
\end{array}\right]=\left[\begin{array}{c}
b\left(\Omega_{1}^{2}+\Omega_{2}^{2}+\Omega_{3}^{2}+\Omega_{4}^{2}\right) \\
b\left(-\Omega_{2}^{2}+\Omega_{4}^{2}\right) \\
b\left(\Omega_{1}^{2}-\Omega_{3}^{2}\right) \\
d\left(-\Omega_{1}^{2}+\Omega_{2}^{2}-\Omega_{3}^{2}+\Omega_{4}^{2}\right) \\
-\Omega_{1}+\Omega_{2}-\Omega_{3}+\Omega_{4}
\end{array}\right] .
$$

The utilized quadrotor's parameters, in equations (1), (2), are listed in Table 1. Table 1

Quadrotor Model Parameters

\begin{tabular}{|c|c|}
\hline$I_{x x}\left(I_{y y}\right)\left[I_{z z}\right]$ & $\mathrm{X}(\mathrm{Y})[\mathrm{Z}]$-axis inertia component \\
$l_{a}$ & Quadrotor Arm length \\
$b(d)$ & Thrust (Drag) coefficient \\
$J_{m}\left(J_{p}\right)$ & Motor (Propeller) Inertia \\
$J_{r}=J_{m}+J_{p} / 4$ & Rotor Inertia \\
\hline
\end{tabular}

The input $U_{1}$ is related to the total thrust, while the inputs $U_{2}, U_{3}, U_{4}$ are related to the rotations of the quadrotor, and $\Omega_{r}$ is the overall residual propeller angular speed. The angles $\eta$ and their derivatives $\dot{\eta}$ are independent of the translational-vector component $(\xi, \dot{\xi})$ the aforementioned system's attitude dynamics in equation (1) can be decoupled from the translational ones (Bouabdallah (2007)), since in general the attitude-subsystem is faster than the translational-subsystem.

The attitude equations correspond to the first six equations of the nonlinear ODE 
in (2). If small perturbations around the operating points $\mathbf{x}^{\circ, \mathbf{j}}=\left[0, \dot{\phi}^{\circ, j}, 0, \dot{\theta}^{\circ, j}, 0, \dot{\psi}^{\circ, j}\right]$ are considered (i.e., $\dot{\phi}=\dot{\phi}^{\circ, j}+\delta \dot{\phi}^{j}$ ), then the PWA linearized attitude equations of motion can be inferred as:

$$
\dot{\mathbf{x}}=\mathbf{A}_{j} \mathbf{x}+\mathbf{B}_{j} \delta \mathbf{u}+\delta \tilde{\mathbf{w}}_{N}
$$

where $\mathbf{x}=\left[\delta \phi^{j}, \delta \dot{\phi}^{j}, \delta \theta^{j}, \delta \dot{\theta}^{j}, \delta \psi^{j}, \delta \dot{\psi}^{j}\right]$ and $\delta \mathbf{u}=\left[\delta U_{1}, \delta U_{2}, \delta U_{3}, \delta U_{4}, \delta \Omega_{r}\right]^{\prime}$, $\delta \tilde{\mathbf{w}_{N}}=\left[0, \delta \tilde{W}_{1}, 0, \delta \tilde{W}_{2}, 0, \delta \tilde{W}_{3}\right]$, and

$$
\mathbf{A}_{j}=\left[\begin{array}{cccccc}
0 & 1 & 0 & 0 & 0 & 0 \\
0 & 0 & 0 & \frac{I_{y y}-I_{z z}}{I_{x x}} \dot{\psi}^{\circ, j} & 0 & \frac{I_{y y}-I_{z z}}{I_{x x}} \dot{\theta}^{\circ, j} \\
0 & 0 & 0 & 1 & 0 & 0 \\
0 \frac{I_{z z}-I_{x x}}{I_{y y}} \dot{\psi}^{\circ, j} & 0 & 0 & 0 & \frac{I_{y y}-I_{x x}}{I_{y y}} \dot{\varphi}^{\circ, j} \\
0 & 0 & 0 & 0 & 0 & 1 \\
0 & \frac{I_{x x}-I_{y y}}{I_{z z}} \dot{\theta}^{\circ, j} & 0 & \frac{I_{x x}-I_{y y}}{I_{z z}} \dot{\varphi}^{\circ, j} & 0 & 0
\end{array}\right]
$$




$$
\mathbf{B}_{j}=\left[\begin{array}{ccccc}
0 & 0 & 0 & 0 & 0 \\
0 & \frac{l_{a}}{I_{x x}} & 0 & 0 & \frac{J_{r}}{I_{x x}} \dot{\theta}^{\circ, j} \\
0 & 0 & 0 & 0 & 0 \\
0 & 0 & \frac{l_{a}}{I_{y y}} & 0 & \frac{J_{r}}{I_{y y}} \dot{\theta}^{\circ, j} \\
0 & 0 & 0 & 0 & 0 \\
0 & 0 & 0 & \frac{1}{I_{z z}} & 0
\end{array}\right] .
$$

\section{Switching Model Predictive Control Scheme}

By considering a $T_{S}$ sampling period, equation (3) can be discretized resulting in a discrete PWA system description:

$$
\mathbf{x}_{d}(k+1)=\mathbf{A}_{j}^{*} \mathbf{x}_{d}(k)+\mathbf{B}_{j}^{*} \delta \mathbf{u}(k)+\tilde{\mathbf{w}}
$$

where $\mathbf{x}_{d}(k) \in \mathscr{X} \subseteq \mathfrak{R}^{6}\left(\delta \mathbf{u}(k) \in \mathscr{U} \in \mathfrak{R}^{5}\right)$ is the state vector (control action), at instant $k T_{S}$. In the general case $j \in \mathscr{S} \triangleq\{1,2, \cdots, s\}$ a finite set of indexes and $s$ denotes the number of affine sub-systems in (6). For polytopic uncertainty, let $\Omega$ be the polytope defined by the convex hull of its vertices $\left[\mathbf{A}_{i}^{*} \mathbf{B}_{i}^{*}\right]$ as $\operatorname{Co}\left\{\left[\mathbf{A}_{1}^{*} \mathbf{B}_{1}^{*}\right], \cdots,\left[\mathbf{A}_{s}^{*} \mathbf{B}_{s}^{*}\right]\right\}$.

The sets $\mathscr{X}$ and $\mathscr{U}$ specify possible state and input constraints and it is assumed that are compact polyhedral sets containing the origin in their interior. Let the set $\mathscr{X}_{i}$ contain the $\mathbf{x}_{i}^{j}$ state, with $i(j)$ related to the specific state (PWA-model), (i.e. $i=2, j=1$ corresponds to the $\dot{\phi}^{\circ}$-variable of the first PWA model). Each element of these vectors satisfies the following bounding inequality:

$$
\mathbf{x}_{i}^{\circ, j} \in \mathscr{X}_{i}: \mathbf{x}_{i}^{\circ, j}-\Delta_{i}=\mathbf{x}_{i}^{\circ, j, \min } \leq \mathbf{x}_{i}^{\circ, j, \max }=\mathbf{x}^{\circ, j} \leq \mathbf{x}^{\circ, j}+\Delta_{i}
$$


where $\Delta_{i} \in \mathfrak{R}^{+}$and $\mathscr{X}=\bigcup \mathscr{X}_{i}, i=1, \ldots, 6$. For the experimental test-cases, the rotation angles are bounded as follows: roll angle $(-\pi / 2<\phi<\pi / 2)$; pitch angle $(-\pi / 2<\theta<\pi / 2)$; and yaw angle $(-\pi<\psi<\pi)$ while the bounds for $\dot{\eta}$ are set to $[-1,1]$.

The control input bounding set $\mathscr{U}$ can be derived by the bounds on the motors' angular velocities $\delta \Omega_{i}, i=1, \ldots, 4$, which are $\delta \Omega_{i} \in\left[0, \delta \Omega_{i}^{\max }\right]$ and by utilizing interval analysis (Moore (1979)). As a result the constraints on the control inputs are formulated as:

$$
\begin{aligned}
& \delta U_{1} \in \mathscr{U}_{1}: \delta U_{1}^{\min }=0 \leq \delta U_{1} \leq b \sum_{i=1}^{4}\left(\delta \Omega_{1}^{\max }\right)^{2}=\delta U_{1}^{\max } \\
& \delta U_{2} \in \mathscr{U}_{2}: \delta U_{2}^{\min }=-b\left(\delta \Omega_{2}^{\max }\right)^{2} \leq \delta U_{2} \leq b\left(\delta \Omega_{4}^{\max }\right)^{2}=\delta U_{2}^{\max } \\
& \delta U_{3} \in \mathscr{U}_{3}: \delta U_{3}^{\min }=-b\left(\delta \Omega_{3}^{\max }\right)^{2} \leq \delta U_{3} \leq b\left(\delta \Omega_{1}^{\max }\right)^{2}=\delta U_{3}^{\max } \\
& \delta U_{4} \in \mathscr{U}_{4}: \delta U_{4}^{\min }=-d\left[\left(\delta \Omega_{1}^{\max }\right)^{2}+\left(\delta \Omega_{3}^{\max }\right)^{2}\right] \leq \delta U_{4} \leq d\left[\left(\delta \Omega_{2}^{\max }\right)^{2}+\left(\delta \Omega_{4}^{\max }\right)^{2}\right]=\delta U_{4}^{\max } \\
& \delta \Omega_{r} \in \mathscr{U}_{5}: \delta \Omega_{r}^{\min }=-\delta \Omega_{1}^{\max }-\delta \Omega_{3}^{\max } \leq \delta \Omega_{r} \leq \delta \Omega_{2}^{\max }+\delta \Omega_{4}^{\max }=\delta \Omega_{r}{ }^{\max }
\end{aligned}
$$

and $\mathscr{U}=\bigcup \mathscr{U}_{i}, i=1, \ldots, 5$. Let the matrix $\mathbf{H}_{i}$ be a zeroed $2 \times 11$ matrix except for its $i$-th column, which is equal to $[1,-1]^{T}$, the the previous constraints can be written in a more compact form as: 


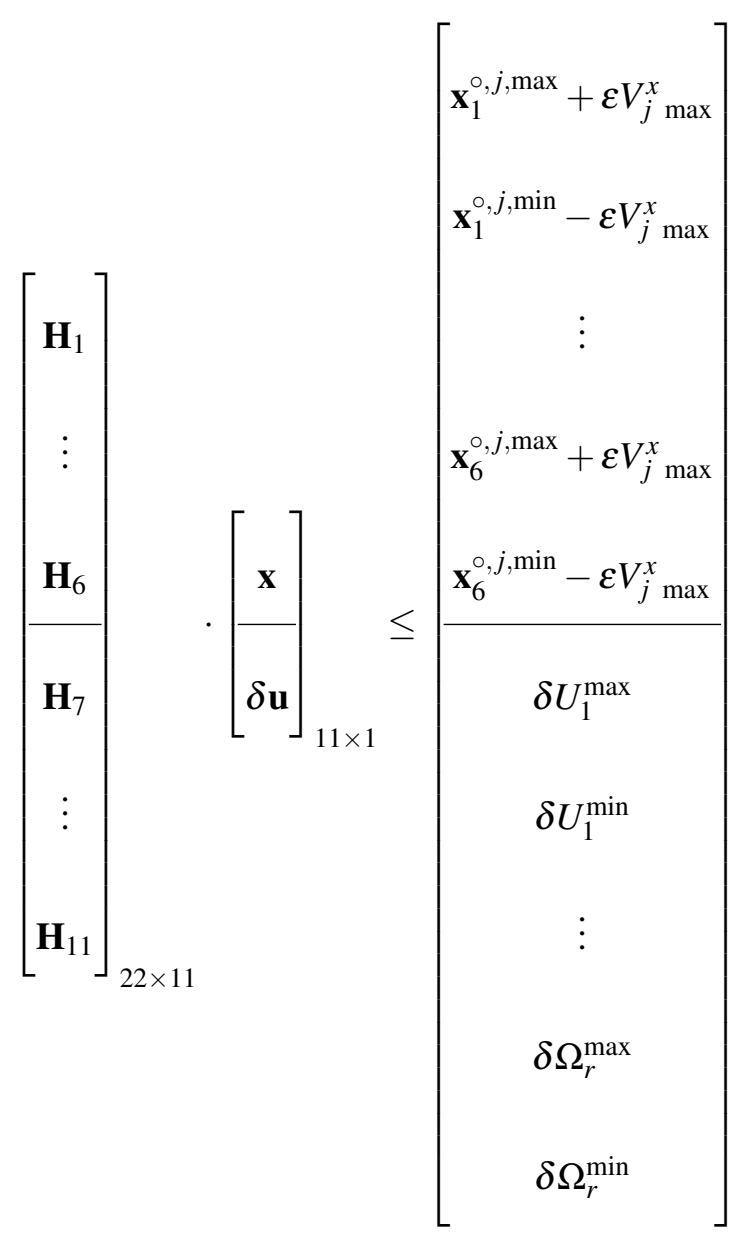

where $\varepsilon$ is a relaxing slack variable and $V_{j \max }^{x}, V_{j \text { min }}^{x}$ are positive constant multipliers for the state constraints which correspond to the 'soft' constraints. These constraints are embedded in the Model Predictive Control computation algorithm in order to compute an optimal controller that counts for the physical and mechanical constraints that restrict the quadrotor's motion.

The $\tilde{\mathbf{w}}$ term corresponds to the effect of the unknown additive disturbance (such as wind-gusts) on the system's dynamics and can be analyzed as $\tilde{\mathbf{w}}=\mathbf{B}_{w} \mathbf{d}(k)$, where $\mathbf{d}(k) \in \mathfrak{R}^{6 \times 1}$ is the vector of unmeasured disturbances that can be modeled as the output of the following linear time invariant system:

$$
\begin{array}{r}
\mathbf{x}_{w}(k+1)=\overline{\mathbf{A}} \mathbf{x}_{w}(k)+\overline{\mathbf{B}} n_{w}(k) \\
\mathbf{d}(k)=\overline{\mathbf{C}} \mathbf{x}_{w}(k)+\overline{\mathbf{D}} n_{w}(k) .
\end{array}
$$


The system described in (9) is driven by random Gaussian noise $\mathbf{n}_{w}(k)$, having zero mean and unit covariance matrix. It should be mentioned that in classical quadrotor-modeling this term is missing (despite its natural inclusion). The relevant bounds for the disturbance $\mathbf{d}$ are assumed to be known and in the work presented in this article, those bounds have been experimentally measured by applying forcible wind gusts and measuring their maximum effect on the quadrotor's attitude.

In a generic framework, the $j$ th switching MP-controller's objective is to optimize the quadratic cost defined in (10), while the state vector is within $\mathscr{X}_{i}$

The model predictive controller for the $j$ th linearized system, is obtained by solving the following optimization problem with respect to the small control commands $\delta \mathbf{u}$ and to the relaxing positive slack variable $\varepsilon$, as

$$
\min _{\delta \mathbf{u}_{j}(k \mid k)} J\left(\delta \mathbf{u}_{j}, \varepsilon\right)
$$

subject to (6), (8), where

$$
\begin{aligned}
J\left(\delta \mathbf{u}_{j}, \boldsymbol{\varepsilon}\right)=\sum_{i=0}^{p-1} & {\left[\mathbf{x}_{d}(k+i+1 \mid k)-\mathbf{r}(k+i+1)\right]^{T} \mathbf{Q}_{j}\left[\mathbf{x}_{d}(k+i+1 \mid k)-\mathbf{r}(k+i+1)\right]+} \\
& \delta \mathbf{u}_{j}(k+i \mid k)^{T} \mathbf{R}_{\delta \mathbf{u}, j} \delta \mathbf{u}_{j}(k+i \mid k)+ \\
& {\left[\mathbf{u}_{j}(k+i \mid k)-\mathbf{u}_{\text {target,j }}(k+i)\right]^{T} \mathbf{R}_{u, j}\left[u(k+i \mid k)-u_{\text {target }, \mathrm{j}}(k+i)+\rho_{e} \varepsilon^{2}\right.}
\end{aligned}
$$

where $p$ is the prediction horizon, $\mathbf{Q}_{\mathbf{j}}, \mathbf{R}_{\delta \mathbf{u}, j}$ and $\mathbf{R}_{\mathbf{u}, j}$ are positive definite matrices of compatible dimensions. Moreover $\mathbf{r}$ corresponds to the reference signal of the controlled system, while no bounds have been considered for the control commands $\delta \mathbf{u}_{\mathbf{j}}$. The weight $\rho_{e}$ on the slack variable $\varepsilon$ penalizes the violation of the constraints, while $\mathbf{u}_{\text {target }, j}(k+i)$ is a setpoint for the input vector. The subscript ()$_{j}$ denotes the $j$ th component of a vector, while $k+i \mid k$ denotes the predicted value of a variable for time $k+i$ based on the information available at the $k$-time instant. 
The control command is computed as $\mathbf{u}_{j}(k)=\mathbf{u}_{j}(k-1)+\delta \mathbf{u}_{j}(k \mid k)^{*}$, where $\delta \mathbf{u}_{j}(k \mid k)^{*}$ is the first element of the resulting optimal sequence. The MPC monitors the weighted sum of controller adjustments: $S_{\delta u}(k)=\sum_{i=1}^{m} \sum_{l=1}^{5}\left\{w_{l}^{\delta u} \delta u_{l}(k+i-1 \mid k)\right\}^{2}$ where $m \leq p$ is the control horizon and $w_{l}^{\delta u}$ is a positive weight; the applied control command is selected in a switching manner according to the current regime of the state vector $\mathbf{x}$. It should be noted that if $s$ increases, then the approximation of the nonlinear system by a large number of linearized systems is more accurate and results in a larger flight-envelope.

As the states $\mathbf{x}_{d}(k), \mathbf{x}_{w}(k)$ may not be directly measurable, their predictions can be obtained based on an extended Kalman state estimator, based on the augmented system description from (6) and (9):

$$
\left[\begin{array}{c}
\mathbf{x}_{d}(k+1) \\
\mathbf{x}_{w}(k+1)
\end{array}\right]=\left[\begin{array}{cc}
\mathbf{A}_{j}^{*} \mathbf{B}_{w} \bar{C} \\
0 & \overline{\mathbf{A}}
\end{array}\right]\left[\begin{array}{c}
\mathbf{x}_{d}(k) \\
\mathbf{x}_{w}(k)
\end{array}\right]+\left[\begin{array}{c}
\mathbf{B}_{j}^{*} \\
0
\end{array}\right] \delta \mathbf{u}(k)+\left[\begin{array}{c}
\mathbf{B}_{w} \overline{\mathbf{D}} \\
\overline{\mathbf{B}}
\end{array}\right] n_{w}(k) .
$$

\section{Experimental Studies}

The proposed control scheme has been applied to the experimental platform of a Draganflyer quadrotor (Srinkanth et al. (2009)), while some modifications have been made in order to increase the quadrotor's capabilities and to allow a computeraided feedback control of the quadrotor's attitude. The programming environment has been National Instruments' LabView running on a Kontron piTX microcontroller (Siagian et. al. (2011)), while the measurement of the quadrotor's attitude state-vector was provided by the utilization of an Xsens MTi-G (Lukianto et al. (2010)) Attitude and Heading Reference System (AHRS), with a wireless (IEEE 
802.15.4) data link between the AHRS and the computer unit. The radio control link between the base station, where the controller resides, and the quadrotor has been achieved by utilizing a National Instruments USB 6229-board, connected with a FUTABA 6EXAP radio transmitter, operating in training mode. The induced wind gust velocities have been measured using an anemometer.

The experimental test-bed is depicted in Figure 2, where the quadrotor has been attached to a Heli-Safe flight test stand modified in order to allow only attitude maneuvers. The attachment point of the quadrotor to the Heli-Safe stand is at the quadrotor's Center of Gravity (CoG) which is on the "equatorial plane" (within a very small tolerance) defined by the helicopter's rotors. In the absence of any wind gust, the quadrotor's Center of Pressure (CoP) is very close to the $\mathrm{CoG}$; given the small size of the quadrotor and the lift of its rotors, results in a smaller effect of the lateral gust flow in comparison to the one reported in (Pounds et al. (2010)).

The wind-gusts are produced using a set of electric fans; the produced near-the-fan wind-gusts are turbulent and after passing them through a pipe-system, presented in Figure 3 the wind-flow affecting the quadrotor is primarily laminar. The windvelocity component $v^{g}$ is expressed with respect to an inertial coordinate frame $x_{0} y_{0} z_{0}$

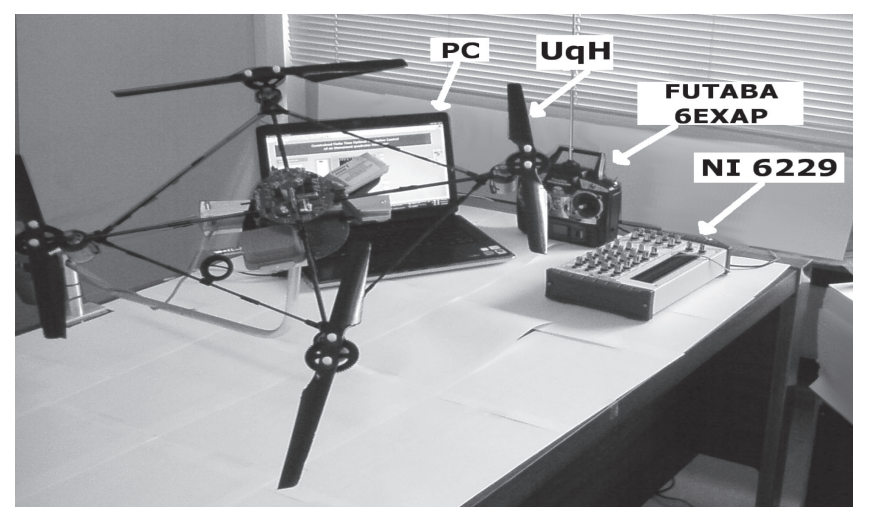

Fig. 2. Quadrotor Attitude Control Experimental Set-Up 




Fig. 3. Artificial Wind-Gust Generating Set-Up

The drag and thrust coefficients of the Draganflyer-quadrotor (Tayebi and McGilvray (2006)), have been verified by the utilization of the Computational Fluid Dynamics XFoil software (Drela M. (1989)). The mass of the modified quadrotor (with the upgraded electronics) has been measured, while the entries of the diagonal inertia matrix, appeared in Table 2, were calculated by utilizing a Finite Element package.

The tuning parameters remained invariant for all switching Model Predictive Controllers have been set as $\mathbf{R}_{\mathbf{u}, j}=10^{4} \cdot I_{5}, \mathbf{R}_{\delta \mathbf{u}, j}=100 \cdot I_{5}$, and $\mathbf{Q}_{j}=1000 \cdot I_{6}$, the prediction horizon $p=10$, the control command horizon $m=2$, while a white noise vector $n_{w}$ was utilized in the controller computation phase. The disturbance vector bounds have been experimentally measured by: a) operating the quadrotor in an open-loop mode with a "hovering" thrust, and b) applying a wind-gust impulse and measuring the maximum rates of the attitude derivatives. Using numerical differentiation, the maximum accelerations of the roll, pitch and yaw angles, which correspond to the maximum additive effect of the wind-gust, have been computed.

For the design and experimental application of the Switching MPC scheme various 
Table 2

Modified Draganflyer Dynamic Parameters

\begin{tabular}{|l|l|l|l|}
\hline Parameter & Description & Value & Units \\
\hline$m_{s}$ & Mass & 0.51 & $\mathrm{~kg}$ \\
$l_{a}$ & arm length & 0.225 & $\mathrm{~m}$ \\
$J_{r}$ & Rotor Inertia & $5.5100 \cdot 10^{-5}$ & $\mathrm{~kg} \cdot \mathrm{m}^{2}$ \\
$I_{x x}$ & Inertia around x-axis & $5.0 \cdot 10^{-3}$ & $\mathrm{~kg} \cdot \mathrm{m}^{2}$ \\
$I_{y y}$ & Inertia around y-axis & $5.0 \cdot 10^{-3}$ & $\mathrm{~kg} \cdot \mathrm{m}^{2}$ \\
$I_{z z}$ & Inertia around z-axis & $8.9 \cdot 10^{-3}$ & $\mathrm{~kg} \cdot \mathrm{m}^{2}$ \\
$b$ & Thrust factor & $2.9 \cdot 10^{-5}$ & $\mathrm{~N} \cdot \mathrm{sec}^{2}$ \\
& & & \\
$d$ & Drag factor & $1.1 \cdot 10^{-6}$ & $\mathrm{~N}-\mathrm{m} \cdot \mathrm{sec}^{2}$ \\
\hline
\end{tabular}

test cases have been considered where the quadrotor model was approximated by 3-PWA systems. In all these cases the sampling period was set to $T_{s}=0.1 \mathrm{sec}$, and the utilized discrete PWA-systems' description has been:

$$
\mathbf{A}_{1}^{*}=\left[\begin{array}{cccccc}
1 & 0.1 & 0 & 0 & 0 & 0 \\
0 & 1 & 0 & 0 & 0 & 0 \\
0 & 0 & 1 & 0.1 & 0 & 0 \\
0 & 0 & 0 & 1 & 0 & 0 \\
0 & 0 & 0 & 0 & 1 & 0.1 \\
0 & 0 & 0 & 0 & 0 & 1
\end{array}\right], \mathbf{B}_{1}^{*}=\left[\begin{array}{cccccc}
0 & 0.178 & 0 & 0 & 0 \\
0 & 3.5593 & 0 & 0 & 0 \\
0 & 0 & 0.178 & 0 & 0 \\
0 & 0 & 3.5593 & 0 & 0 \\
0 & 0 & 0 & 0.5682 & 0 \\
0 & 0 & 0 & 11.3636 & 0
\end{array}\right]
$$

for $-0.05<\dot{\phi}^{\circ, j}, \dot{\theta}^{\circ, j}, \dot{\psi}^{\circ, j}<0.05$, 


$$
\mathbf{A}_{2}^{*}=\left[\begin{array}{cccccc}
1 & 0.1 & 0 & -0.0001 & 0 & -0.0001 \\
0 & 1 & 0 & -0.0025 & 0 & -0.0025 \\
0 & 0.0001 & 1 & 0.1 & 0 & 0.0001 \\
0 & 0.0025 & 0 & 1 & 0 & 0.0025 \\
0 & 0 & 0 & 0 & 1 & 0.1 \\
0 & 0 & 0 & 0 & 0 & 1
\end{array}\right], \mathbf{B}_{2}^{*}=\left[\begin{array}{cccccc}
0 & 0.178 & -0.0001 & -0.0005 & 0 \\
0 & 3.5593 & -0.0044 & -0.0140 & 0.0001 \\
0 & 0.0001 & 0.178 & 0.0005 & 0 \\
0 & 0.0044 & 3.5593 & 0.014 & 0.0001 \\
0 & 0 & 0 & 0.5682 & 0 \\
0 & 0 & 0 & 11.3636 & 0
\end{array}\right]
$$

for $0.05<\dot{\phi}^{\circ, j}, \dot{\theta}^{\circ, j}, \dot{\psi}^{\circ, j}<0.15$, and

$$
\mathbf{A}_{3}^{*}=\left[\begin{array}{cccccc}
1 & 0.1 & 0 & 0.0001 & 0 & 0.0001 \\
0 & 1 & 0 & 0.0025 & 0 & 0.0025 \\
0 & -0.0001 & 1 & 0.1 & 0 & -0.0001 \\
0 & -0.0025 & 0 & 1 & 0 & -0.0025 \\
0 & 0 & 0 & 0 & 1 & 0.1 \\
0 & 0 & 0 & 0 & 0 & 1
\end{array}\right], \mathbf{B}_{3}^{*}=\left[\begin{array}{cccccc}
0 & 0.178 & 0.0001 & 0.0005 & 0 \\
0 & 3.5593 & 0.0044 & 0.014 & -0.0001 \\
0 & -0.0001 & 0.178 & -0.0005 & 0 \\
0 & -0.0044 & 3.5593 & -0.014 & -0.0001 \\
0 & 0 & 0 & 0.5682 & 0 \\
0 & 0 & 0 & 11.3636 & 0
\end{array}\right]
$$

for $-0.15<\dot{\phi}^{\circ, j}, \dot{\theta}^{\circ, j}, \dot{\psi}^{\circ, j}<-0.05$. The constraints on the inputs have been computed based on the physical parameters of the system and specifically: a) the maximum angular velocity of the motor/gearbox , b) the thrust factor, and c) the drag factor. Based on the above values the following constraints on the inputs could be realized as $0 \leq U_{1} \leq 11.23,\left|U_{2}\right| \leq 5.61,\left|U_{3}\right| \leq 5.61,\left|U_{4}\right| \leq 0.16$. The state constraints have been selected as $\left|\mathbf{x}_{i}^{\circ, j}\right| \leq \pi / 2 \mathrm{rad}, i=1,3,5$, and $\left|\mathbf{x}_{i}^{\circ, j}\right| \leq 1 \mathrm{rad} / \mathrm{sec}$, $i=2,4,6$

In order to study the characteristics and experimentally verify the capabilities of the described Switching MPC (SMPC) scheme, an extended set of experimental results are being presented. In Figures 5 through 13, the reference signal is depicted in dashed-black line, while the SMPC-quadrotor's response is presented with solid black line. In these Figures, it is also presented the quadrotor's response 
for the following cases: a) PID-control (red line), and b) LQ-control (blue-line). The weighting matrices employed in the the cost:

$$
J=\int_{0}^{\infty}\left(\hat{\mathbf{x}}^{T} Q_{L Q} \hat{\mathbf{x}}+\delta \mathbf{u}^{T} R_{L Q} \delta \mathbf{u}\right) d t
$$

of the LQ-controller have been $Q_{L Q}=5000 * \operatorname{diag}(2,1,2,1,2,1)$ and $R_{L Q}=100 *$ $\mathbf{I}_{5 \times 5}$, while the adopted system description corresponded to the hovering case. The PID-controller parameters have been tuned in an ad-hoc manner in order to provide a fast rise time, while at the same time avoid saturating the control inputs.

It should be noted that the roll, pitch and yaw angles of the quadrotor are not directly measured and an Extended Kalman Filter (EKF) has been employed as presented in Figure 4. The IMU-sensors provide the translational accelerations $\ddot{x}, \ddot{y}, \ddot{z}$, which coupled to the angular velocities $\dot{\phi}, \dot{\psi}, \dot{\theta}$ and the magnetometer's readings $m_{x}, m_{y}, m_{z}$, through an Extended Kalman Filter, provide an estimate of the roll, pitch and yaw angles; these estimates (rather than the actual measurements) have been utilized in the control algorithms (Bristeau et al. (2010)).

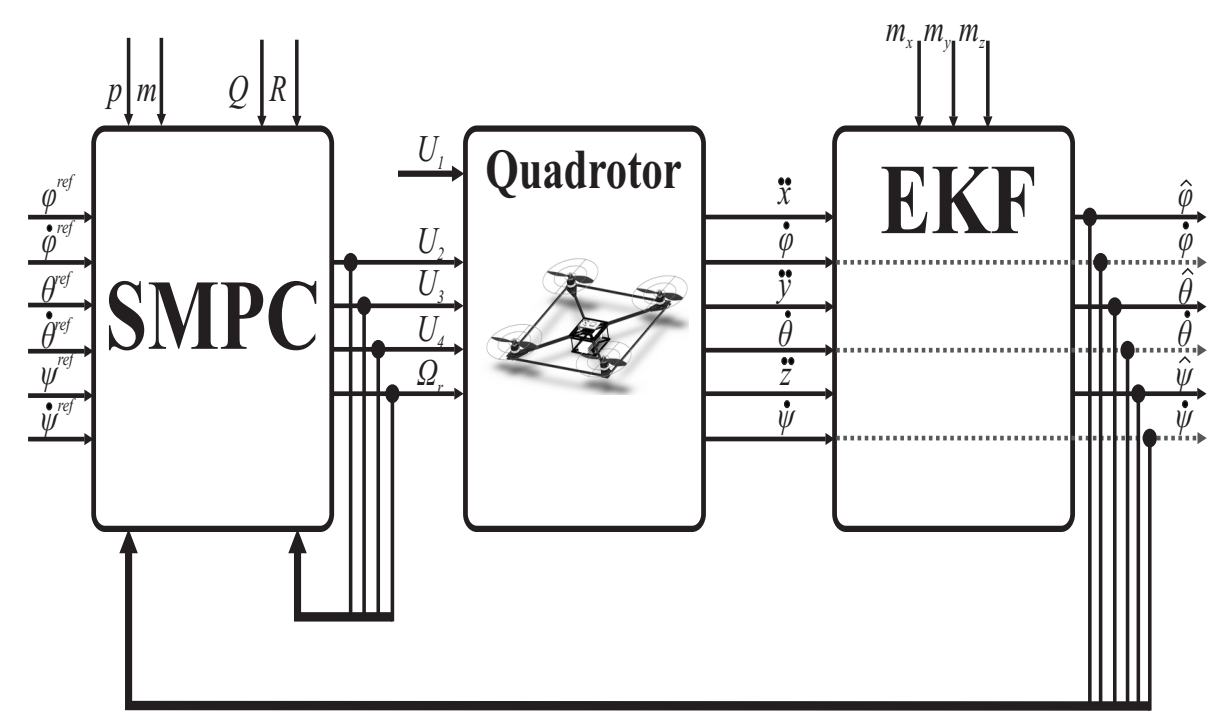

Fig. 4. Switched Model Predictive Controller/Identification Structure 


\subsection{Attitude Regulation and Setpoint SMPC Quadrotor Response}

In the first presented test-case the efficiency of the proposed control scheme has been evaluated in an output regulation problem, for the cases: a) without the impact of wind-gusts, and b) in the presence of wind-gusts. The attitude subsystem has been configured to perform regulation at $\mathbf{r}=\mathbf{0}_{6 \times 1}$ and the experimental responses are presented in Figure 5. The SMPC quadrotor accurately achieved regulation in the absence of wind-gusts and has presented significant robustness against the effects of a forcible wind with a velocity vector $\mathbf{v}^{g}=\left[v_{x}^{g}, v_{y}^{g}, v_{x}^{g}\right]^{T}=$ $[1.31,3.84,1.65]^{T} \mathrm{~m} / \mathrm{s}$, which corresponds to three in the Beaufort scale.

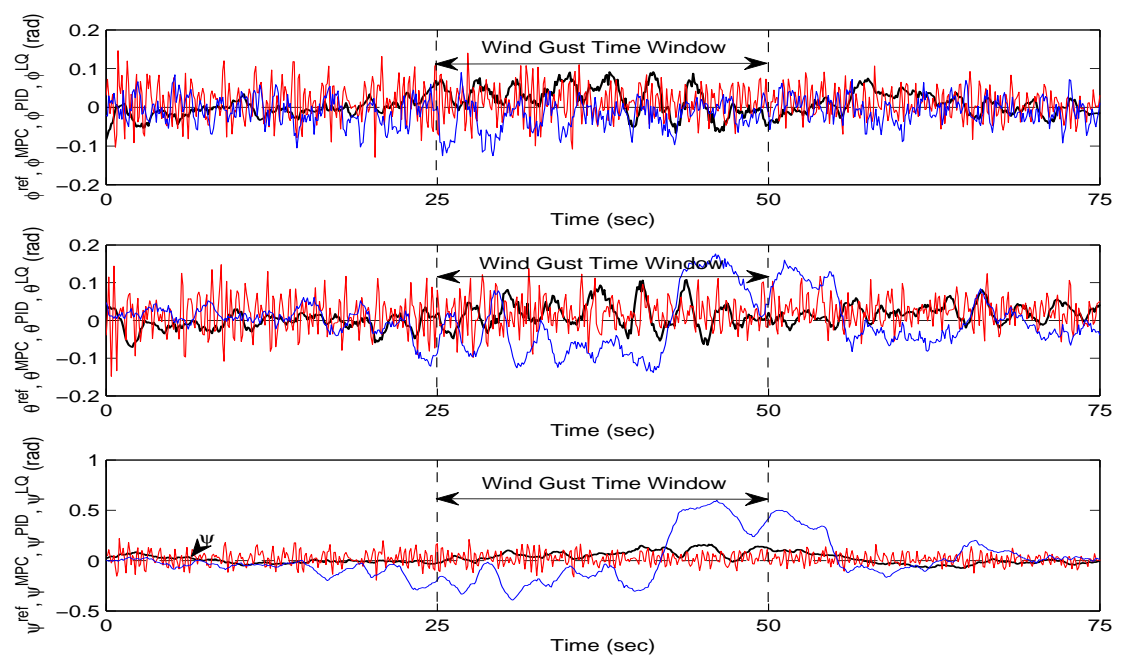

Fig. 5. Quadrotor's regulation response in the absence of wind gusts and inside a $\mathrm{x}(1.31 \mathrm{~m} / \mathrm{s}), \mathrm{y}(3.84 \mathrm{~m} / \mathrm{s})$ and $\mathrm{z}(1.65 \mathrm{~m} / \mathrm{s})$ directional Wind Gust

Under the influence of the same environmental situations, the performance of SMPC has been also evaluated for the case of a setpoint at $\mathbf{r}=[-0.0873 \mathrm{rad}, 0,0.175 \mathrm{rad}, 0$, $0.349 \mathrm{rad}, 0]$ as depicted in Figure 6. From the results obtained, it is evident that the controller effectively maintains the outputs in the neighborhood of the desired setpoint. It should be also noted that in this case, the influence of the wind-gusts is greater for the reasons that: a) the setpoint convergence is harder to be achieved, 
and b) the significant increase in the aerodynamic effects due to the rotation of the quadrotor, and the relative effects of wind-gust.

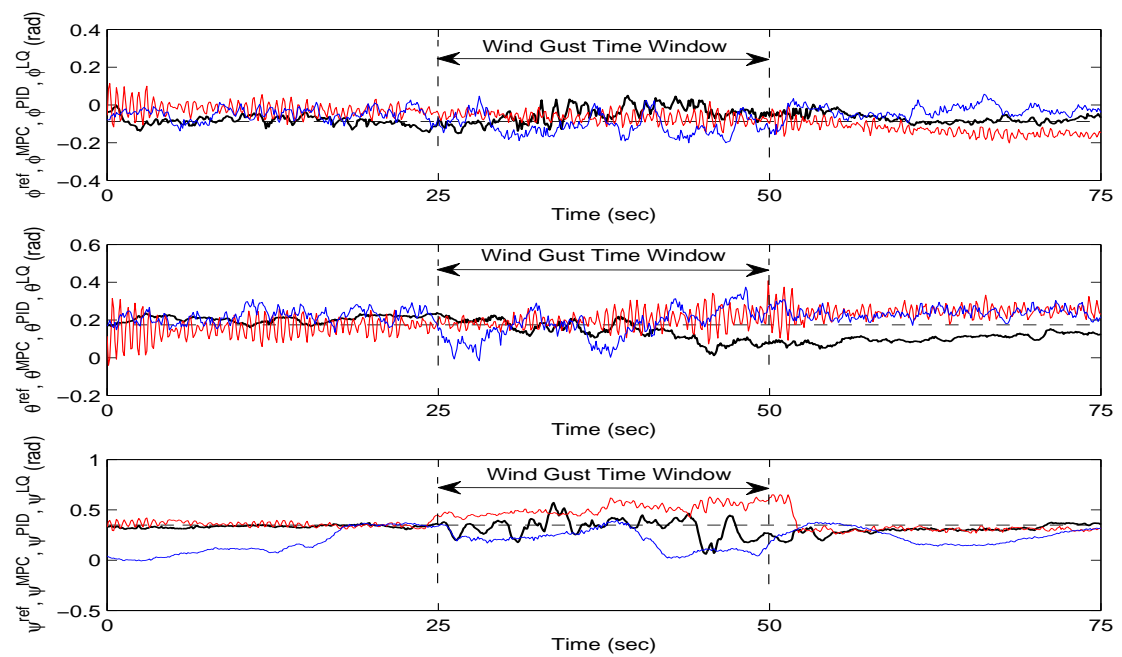

Fig. 6. Quadrotor's setpoint $(\mathbf{r}=[-0.0873 \mathrm{rad}, 0,0.175 \mathrm{rad}, 0,0.349 \mathrm{rad}, 0])$ response in the absence of wind gusts and inside a $x(1.31 \mathrm{~m} / \mathrm{s}), \mathrm{y}(3.84 \mathrm{~m} / \mathrm{s})$ and $\mathrm{z}(1.65 \mathrm{~m} / \mathrm{s})$ directional Wind Gust

\subsection{Attitude Tracking SMPC Quadrotor Response}

In order to evaluate the performance of the developed controller at aggressive maneuvers, the following periodic signal ( $50 \mathrm{sec}$ period) has been applied:

$$
\begin{aligned}
\phi^{r e f}(t) & =0.08732 \operatorname{sign}(t-25) \mathrm{rad} \\
\theta^{r e f}(t) & =-0.175 \operatorname{sign}(t-25) \mathrm{rad} \\
\psi^{r e f}(t) & =0.0175(1-\operatorname{sign}(t-25)) \mathrm{rad}
\end{aligned}
$$

The responses in the absence (presence) of forcible wind gusts are being depicted in Figure 7 (Figure 8). The controller manages to accurately track the desired reference pulse, while providing fast and precise responses, and effectively attenuate the forcible, constant and of high speed wind. 
The small steady state error is being related to the wind's blowing direction. In general, the response is influenced by: a) the angle between the rotors' plane and the wind-velocity vector, and b) the relative yaw angle (since it affects the rotor that is being first influenced by the wind-gust).
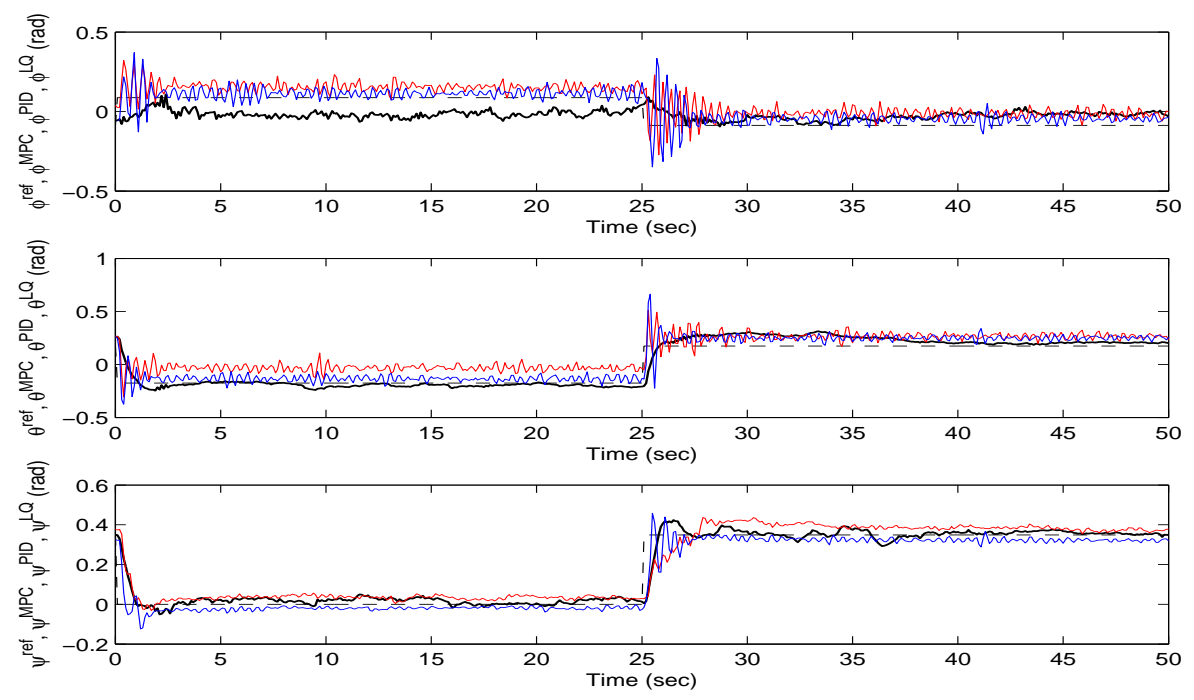

Fig. 7. Quadrotor's response for the pulse-wave tracking problem in the absence of wind gusts
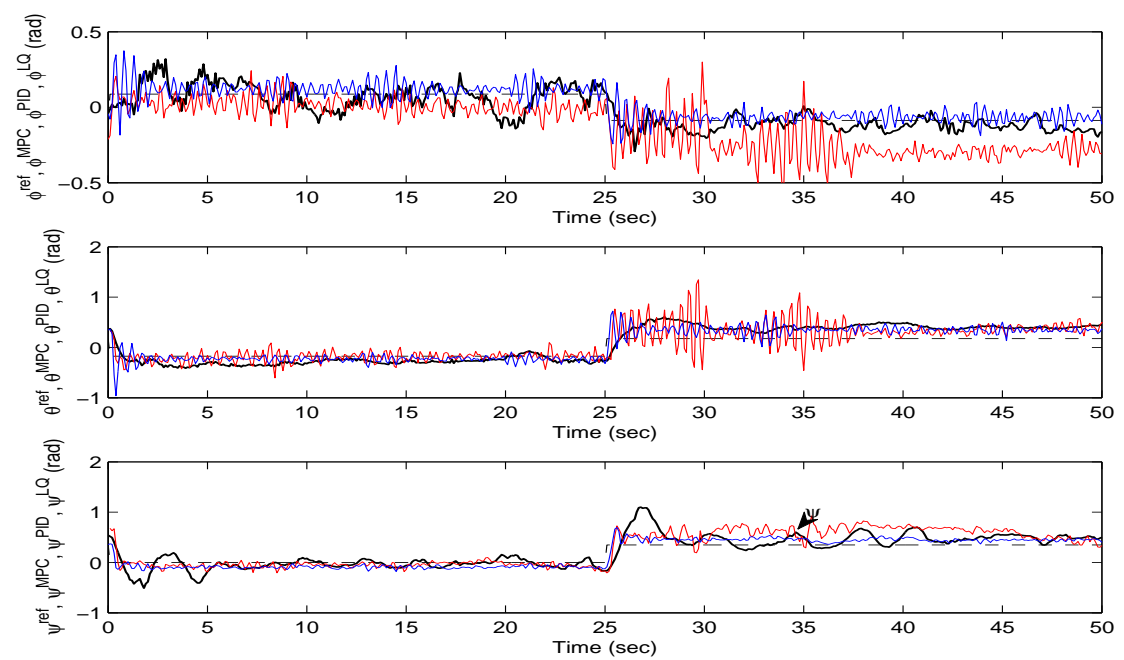

Fig. 8. Quadrotor's response for the pulse-wave tracking problem inside a $\mathrm{x}(1.31 \mathrm{~m} / \mathrm{s})$, $\mathrm{y}(3.84 \mathrm{~m} / \mathrm{s})$ and $\mathrm{z}(1.65 \mathrm{~m} / \mathrm{s})$ directional wind gust

Moreover, the performance of the proposed control scheme has been also evaluated 
for sinusoidal references. More specifically, the response of the SMPC quadrotor has been tested for: a) both in the case of a sinusoidal reference at yaw, while performing regulation at roll and pitch, and b) for the case of sinusoidal references for all the roll, pitch, yaw motions. In the first case, the reference has been set as $[\phi, \theta, \psi]=[0.349 \sin (0.1257 t), 0,0]$ and the results with and without the presence of winds are being presented in Figures 9 and 10 respectively.
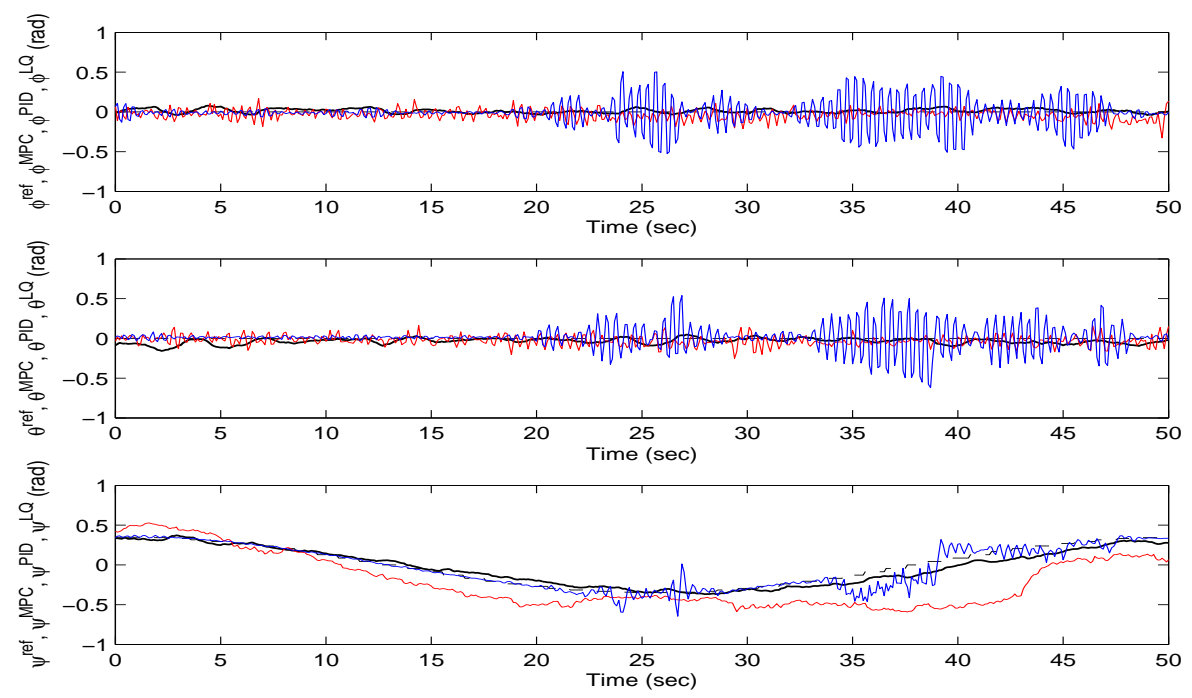

Fig. 9. Quadrotor's response for reference inputs $[\phi, \theta, \psi]=[0.349 \sin (0.1257 t), 0,0] \mathrm{rad}$ in the absence of wind gusts

In the second test case the reference signals have been selected as:

$$
[\phi, \theta, \psi]=[-0.175 \sin (0.1257 t),-0.175 \sin (0.1257 t), 0.349 \sin (0.1257 t)] \mathrm{rad}
$$

The results in the absence of wind disturbances are being presented in Figure 11, while the results with constant wind are being presented in Figure 12. In addition, the results for the case of a sudden forcible wind-gust are being depicted in Figure 13.

The obtained oscillations in the experimental response, when forcible wind-gusts have been applied, are related mainly to: a) the selected slow sampling rate, and 

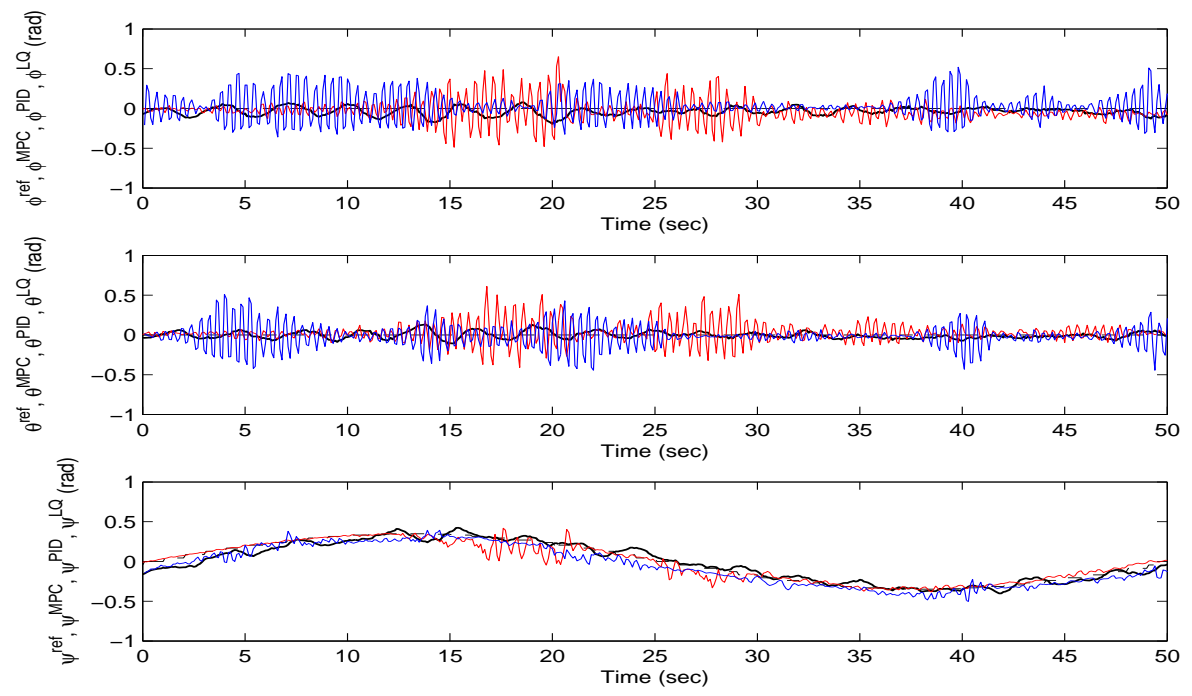

Fig. 10. Quadrotor's response for reference inputs $[\phi, \theta, \psi]=[0.349 \sin (0.1257 t), 0,0] \mathrm{rad}$ inside a $x(1.31 \mathrm{~m} / \mathrm{s}), y(3.84 \mathrm{~m} / \mathrm{s})$ and $\mathrm{z}(1.65 \mathrm{~m} / \mathrm{s})$ directional wind gust
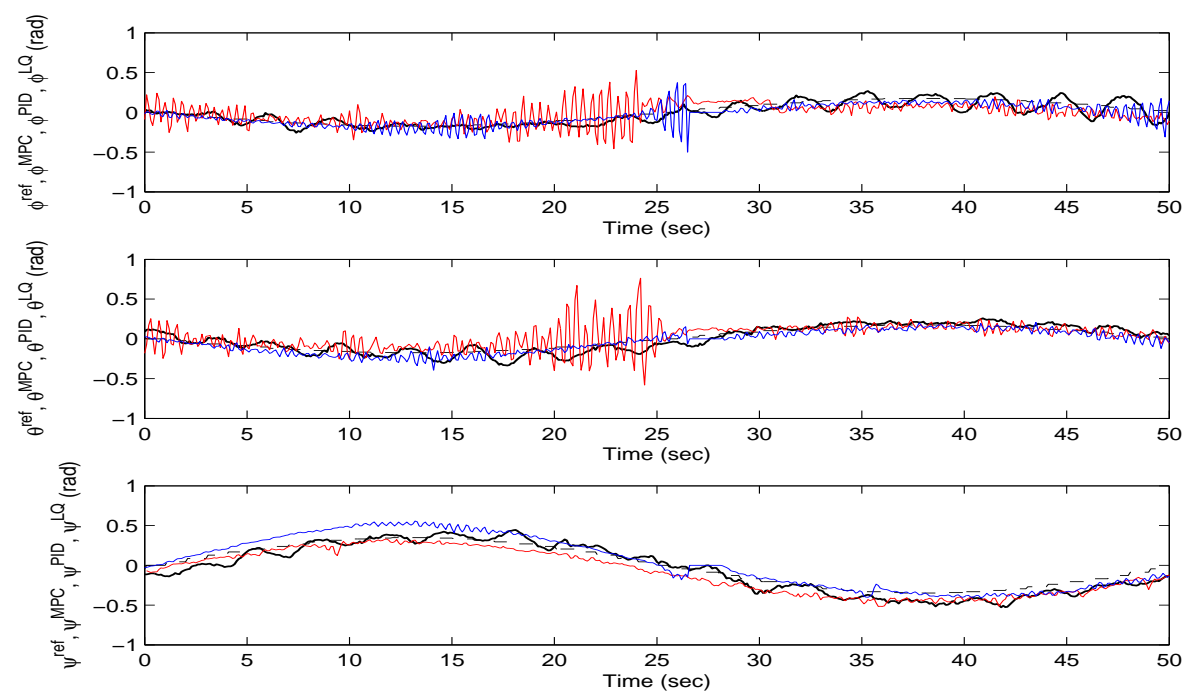

Fig. 11. Quadrotor's response for sinusoidal reference inputs $[\phi, \theta, \psi]=[-0.175 \sin (0.1257 t),-0.175 \sin (0.1257 t), 0.349 \sin (0.1257 t)] \operatorname{rad}$ in the absence of wind gusts

b) the asymmetric forces produced by the different lift components, between the leading and trailing rotors (Hoffmann et al. (2007),Pounds et al. (2010)). It should be noted that the second cause is not the dominant one, due to the small size and the lift of the rotors. However, for the case of large quadrotors this effect is quite 
important as it has been observed in (Pounds et al. (2010)).
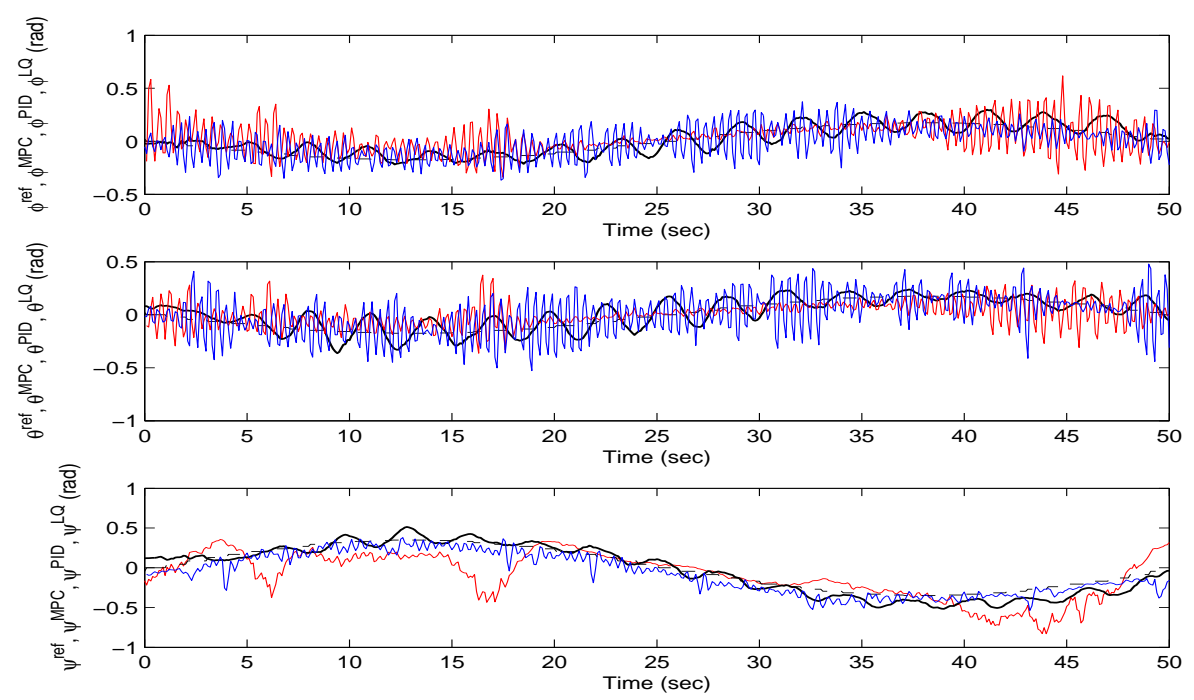

Fig. 12. Quadrotor's response for the case of sinusoidal references inside a $\mathrm{x}(1.31 \mathrm{~m} / \mathrm{s})$, $\mathrm{y}(3.84 \mathrm{~m} / \mathrm{s})$ and $\mathrm{z}(1.65 \mathrm{~m} / \mathrm{s})$ directional wind gust
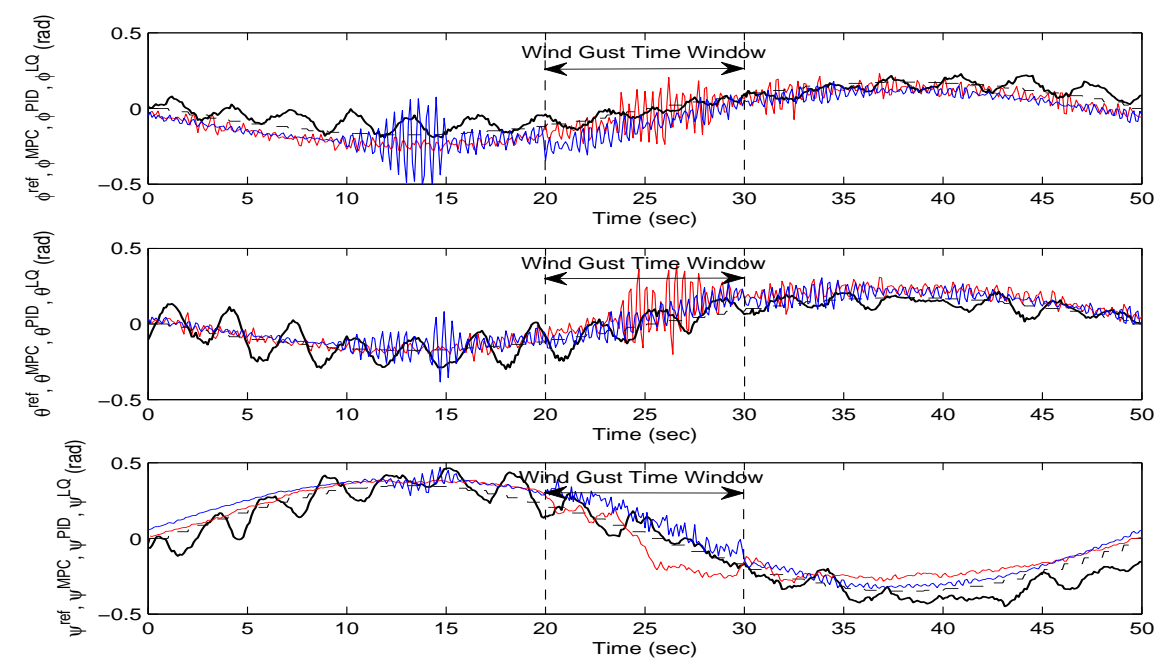

Fig. 13. Quadrotor's response for the case of sinusoidal references at the presence of a $10 \mathrm{sec}$ sudden $\mathrm{x}(1.8 \mathrm{~m} / \mathrm{s}), \mathrm{y}(4.02 \mathrm{~m} / \mathrm{s})$ and $\mathrm{z}(1.9 \mathrm{~m} / \mathrm{s})$ directional wind gust

A comparison of the mean square error (MSE)

$$
\left(\frac{T_{S}}{50}+1\right) \sum_{i=0}^{\frac{50}{T_{S}}}\left\{\left[\phi^{r e f}\left(i T_{s}\right)-\hat{\phi}\left(i T_{S}\right)\right]^{2}+\left[\theta^{r e f}\left(i T_{S}\right)-\hat{\theta}\left(i T_{S}\right)\right]^{2}+\left[\psi^{r e f}\left(i T_{S}\right)-\hat{\psi}\left(i T_{S}\right)\right]^{2}\right\}
$$

between the SMPC, PID and LQ control, for the previous test-cases, is being 
presented in Figure 14, where in most of the cases, given the large sampling pe$\operatorname{riod}\left(T_{S}=0.1 \mathrm{sec}\right)$, the SMPC-controlled system has superior response, if compared with the PID/LQ control scheme . When the sampling period decreases (i.e., $T_{s}=10 \mathrm{msec}$ ), the SMPC still outperforms the PID/LQ control scheme with a slight margin.

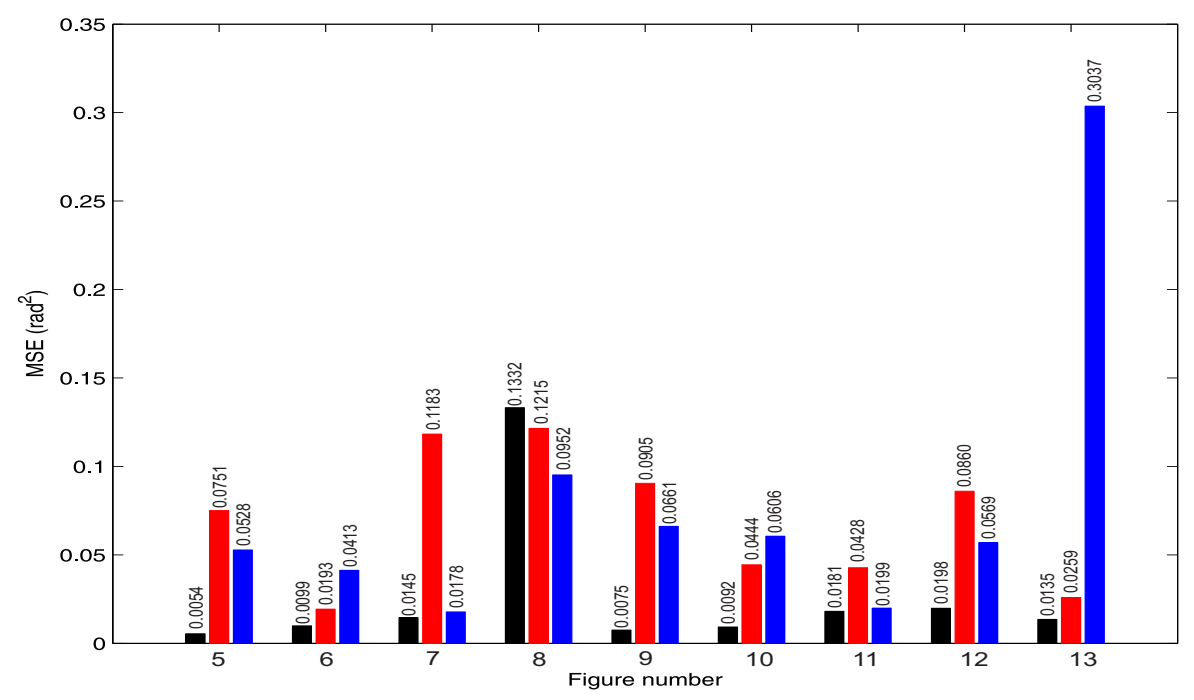

Fig. 14. MSE Quadrotor Response Comparison (SMPC (black) vs. PID (red) vs. LQ (blue))

It should be emphasized that the ad-hoc parameters' selection for the prediction and control horizon, $(p, m)=(10,2)$, that have been utilized in the formulation of the MPC-cost (10) have not been the optimal ones, as highlighted in Figure 15, where the achieved MSE for the adopted SMPC is depicted for various $p, m$ parameters; the optimal parameter-pair corresponds to $(p, m)=(14,4)$. Since the computational burden monotonically increases with $p$ and $m$, smaller values are generally preferred; however in general these small values typically lead to an excitation of the high frequency harmonics and the quadrotor's response oscillates significantly. 


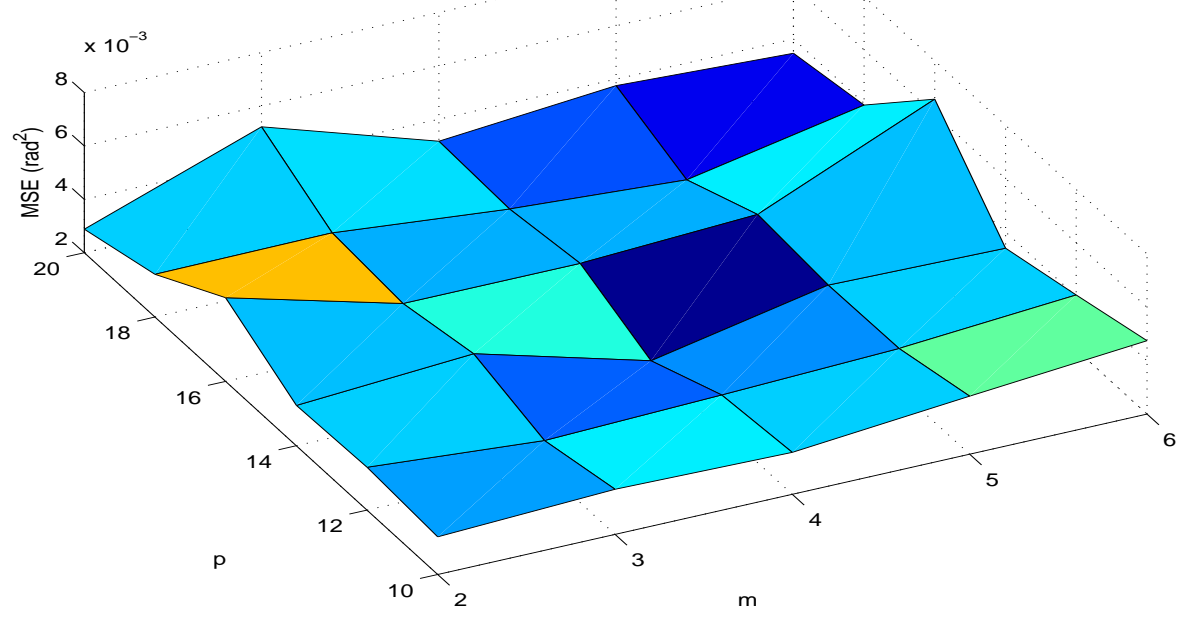

Fig. 15. SMPC Performace w.r.t. various $p$ and $m$ Parameters

\subsection{Attitude Regulation of a SMPC free-flying Quadrotor at High Sampling Rate}

In order to examine the response of a free-flying quadrotor at high sampling rates, additional experimental studies have been performed. In Figure 16 the attitude regulation of the free-flying quadrotor is being presented for $T_{S}=0.01 \mathrm{sec}$ at the absence of wind-gusts.


Fig. 16. Free-Flying Quadrotor Attitude Regulation Response for $T_{s}=0.01 \mathrm{sec}$

It should be noted that the aerodynamic effects, coupled to the small sampling rate 
$(10 \mathrm{~Hz})$ of the previous case were the dominant factors affecting the quadrotor's performance. When the rate was increased (at $100 \mathrm{~Hz}$ ) for the free-flying (not attached at the Heli-safe) helicopter, a significant reduction in the pitching and rolling magnitude was observed; this is an indication that the controller's performance is primarily being influenced by the utilized sampling rate while being tolerant to the aerodynamic effects.

The efficiency of the modified controller has also been verified in the case of applying wind-gusts to the free flying quadrotor and the corresponding attitude regulation response is depicted in Figure 17.
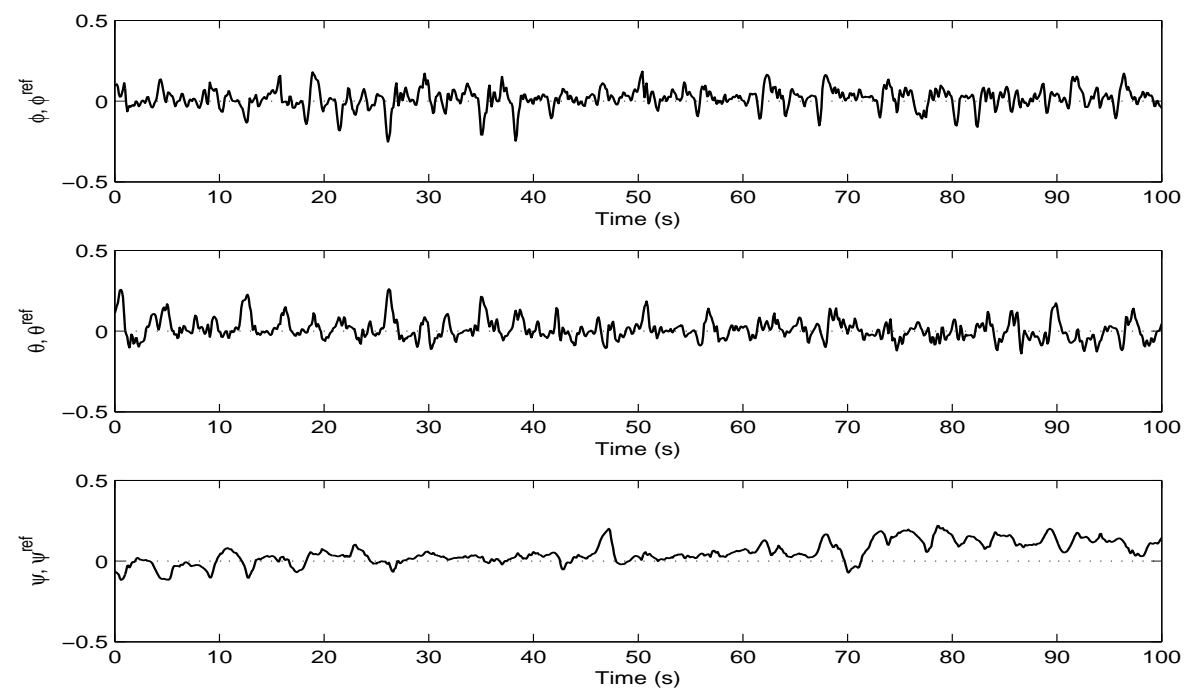

Fig. 17. Free-flying Quadrotor Attitude Regulation Response for $T_{s}=0.01 \mathrm{sec}$ subject to a $\mathrm{x}(1.48 \mathrm{~m} / \mathrm{s}), \mathrm{y}(2.69 \mathrm{~m} / \mathrm{s})$ and $\mathrm{z}(1.56 \mathrm{~m} / \mathrm{s})$ directional Wind Gust

Overall, the proposed SMPC-structure is a promising solution for the quadrotor control problem, since in comparison with existing techniques it takes into account: a) the actuator saturation constraints, b) the effect of disturbances in the controller design phase, and c) the larger flight envelope as a result of the multiple linearization points. Issues that remain open for further research include: 1) the selection of the control and prediction horizons, where large values typically lead to a smoother 
yet slower response, 2) the selection of the number for the utilized PWA-models, the appropriate linearization operating points and the bounds for each region, 3) the need to have an accurate knowledge of the quadrotor dynamics, 4) the effect the noise and/or bias in the measurements,since these may affect the performance of the EKF, and 5) the assumption of a white-noise kernel for the induced windgust.

\section{Conclusions}

In this paper, a Switching Model Predictive Controller for an Unmanned quadrotor Helicopter flying in an environment at the presence of wind-gusts has been presented. The main contribution of the proposed control scheme includes the development of a model predictive controller, for the set of the induced linearized Piecewise Affine models of the system. The PWA-approximations cover a larger subset of the quadrotor's flight envelope, and the controller takes into account the physical constraints of the system. Finally the controller has been experimentally shown sufficient robustness against wind-gust disturbances, whose bounds have been utilized for the off-line computation of the controller. The presented experimental results indicate the high overall efficiency of the proposed SMPC-scheme in attitude maneuvers tracking, with wind-gust disturbance attenuation.

\section{References}

Alexis, K., Nikolakopoulos, G., Tzes, A., 2010a. Constrained optimal attitude control of a quadrotor helicopter subject to wind-gusts: Experimental studies. In: 
2010 American Control Conference, pp. 4451-4455, June 30 - July 2, Baltimore, USA.

Alexis, K., Nikolakopoulos, G., Tzes, A., 2010b. Design and experimental verification of a constrained finite time optimal control scheme for the attitude control of a quadrotor helicopter subject to wind gusts. In: 2010 International Conference on Robotics and Automation, pp. 1636-1641, May 3-8, Anchorage, Alaska, USA.

Guerrero-Castellanos, J. F., Marchand, N., Hably, A., Lesecq, S. and Delamare, J., 2011. Bounded attitude control of rigid bodies: Real-time experimentation to a quadrotor mini-helicopter. In Control Engineering Practice (In press), doi:10.1016/j.conengprac.2011.04.004.

Alexis, K., Nikolakopoulos, G., Tzes, A., 2010c. Model Predictive Control for a Miniature Coaxial Helicopter. In: 7th IFAC Symposium on Intelligent Autonomous Vehicles, article \#16094, vol. 7, no. 1, September 6-8, Lecce, Italy.

Alexis, K., Nikolakopoulos, G., Tzes, A., Dritsas, L., June 2009. Coordination of helicopter UAVs for aerial Forest-Fire surveillance. In: Applications of Intelligent Control to Engineering Systems. Springer Netherlands, pp. 169-193.

S. Bouabdallah, 2007. Design and Control of quadrotors with application to autonomous flying, Ph.D. dissertation, EPFL STI School of Engineering 2007. Lausanne, Switzerland.

Bouabdallah, S., Becker, M., Siegwart, R., 2007. Autonomous miniature flying robots: coming soon! - research, development, and results. In IEEE Robotics \& Automation Magazine, 14 (3), 88-98.

Bouabdallah, S., Noth, A., Siegwart, R., 2004. PID vs LQ control techniques applied to an indoor micro quadrotor. In: Proceedings of the IEEE/RSJ International Conference on Intelligent Robots and Systems, pp. 2451-2456, September 28 - October 2, Sendai, Japan. 
Bouabdallah, S., Siegwart, R., 2007. Full control of a quadrotor. In: 2007 IEEE/RSJ International Conference on Intelligent Robots and Systems. pp. 153-158, October 29 - November 1, San Diego, CA, USA,

Bristeau, P-J., Martin, P., Salaun, E., and Petite N., 2009. The Role of Propeller Aerodynamics in the Model of a Quadrotor UAV, In European Control Conference, pp. 683-688, August 23-26, Budapest, Hungary.

Costello, M. F., 1992. A theory of the analysis of rotorcraft operation in atmospheric turbulence. Ph.D. dissertation, School of Aerospace Engineering, Georgia Institute of Technology.

Drela M. 1989, XFOIL: an analysis and design system for low Reynolds number airfoils. In: Mueller TJ, Editor. Low Reynolds numbers aerodynamics, Lecture Notes in Engineering, vol. 54. pp. 1-12, New York: Springer, 1989.

Bristeau, P.-J., Dorveaux, E., Vissiere, D., Petit, N., 2010. Hardware and software architecture for state estimation on an experimental low-cost small-scaled helicopter. In Control Engineering Practice, vol. 18, No. 7., pp. 733-746.

Pounds P., Mahony R., and P. Corke 2010. Modelling and control of a large quadrotor robot. In: Control Engineering Practice, Special Issue on Aerial Robotics, vol. 18, pp. 691-699.

Hoffmann, G. M., Huang, H., Waslander, S. L., Tomlin, C. J., 2007. Quadrotor helicopter flight dynamics and control: Theory and experiment. In: Proc. of the AIAA Guidance, Navigation, and Control Conference, article 6461, August 2023, Hilton Head, SC, USA.

Kondak, K., Bernard, M., Meyer, N., Hommel, G., 2007. Autonomously flying VTOL-Robots: modeling and control. In: 2007 IEEE International Conference on Robotics and Automation. pp. 736-741, April 10-14, Rome, Italy

Lukianto C., Honniger C, Sternberg H., 2010, Pedestrian Smartphone-Based Indoor Navigation Using Ultra Portable Sensory Equipment. In: Proceedings of 
the IEEE 2010 International Conference on Indoor Positioning and Navigation, pp. 1-5, September 15-17, Zurich, Switzerland.

Martini, A. and Léonard, F. and Abba, G. 2009. Dynamic Modelling and Stability Analysis of Model-Scale Helicopters Under Wind Gust. In: Journal of Intelligent Robotic Systems 2009, pp. 647-686, Kluwer Academic Publishers, Hingham, MA, USA.

Merino, L., Caballero, F., Martinez, J., Ollero, A., 2005. Cooperative fire detection using unmanned aerial vehicles. In Proceedings of the IEEE International Conference on Robotics and Automation, pp. 1884-1889, April 18-22, Barcelona, Spain.

Metni, N., Hamel, T., November 2007. A UAV for bridge inspection: Visual servoing control law with orientation limits. Automation in Construction 17 (1), $3-10$.

Moore, R., 1979. Methods and Applications of Interval Analysis. SIAM Bookmart, Philadelphia.

Perhinschi M. G. 1998. A Model of Atmospheric Turbulence for Rotorcraft Simulation and Analysis of Stability and Performance. In: 1998 AIAA Modeling and Simulation Technologies Conference, pp. 635-642, Boston, MA, U.S.A.

Pounds P., Mahony R., and Corke P.,2006, Modelling and control of a quad-rotor robot, Proceedings of the Australian Conference on Robotics and Automation, December 6-8, Auckland, New Zealand.

Pounds P., Mahony R., and P. Corke 2010. Modelling and control of a large quadrotor robot. In: Control Engineering Practice, Special Issue on Aerial Robotics, vol. 18, pp. 691-699.

Raffo, G., Ortega, M., Rubio, F., 2010. An integral predictive/nonlinear control structure for a quadrotor helicopter. Automatica 46 (1), 29 - 39.

Siagian C., Chang C-K., Voorhies R., Itti L. 2011. Beobot 2.0: Cluster architecture 
for mobile robotics, In Journal of Field Robotics Volume 28, Issue 2, pp 278302. Srinkanth M., Dydek Z., Annaswamy A., Lavretsky E., 2009. A Robust Environment for Simulation and Testing of Adaptive Control for Mini-UAVs. In Proceedings of the 2009 American Control Conference, pp. 5398-5403, June 10-12, St. Louis, MO, USA.

Tayebi, A., McGilvray, S., 2006. Attitude stabilization of a VTOL quadrotor aircraft. IEEE Transactions on Control Systems Technology 14 (3), 562-571.

Waslander, S. L., Hoffmann, G. M., Jang, J. S., Tomlin, C. J., 2005. Multi-agent quadrotor testbed control design: Integral sliding mode vs. reinforcement learning. In: Proceedings of the IEEE/RSJ International Conference on Intelligent Robotics and Systems, pp. 468-473, August 2-6, Edmonton, Alberta, Canada

Yang, X., Pota, H., Garrat, M., July 2009. Design of a gust-attenuation controller for landing operations of unmanned autonomous helicopters. In: 18th IEEE International Conference on Control Applications. Saint Petersburg, Russia, pp. $1300-1305$. 